\title{
III.
}

Aus der Klinik des Herrn Prof. M. W. Janowsky in Petersburg.

\section{Ueber das Verhalten der roten Blutkörperchen zum Wechselstrom.}

Von

Dr. A. F. Drschewetzky.

Über das Verhältnis der roten Blutkörperchen und speziell über ihre Resistenz dem elektrischen Strom gegenüber liegen Experimente von A. Rollett vor, der als erster den Wechselstrom zur Untersuchung des Blutes anwendete. In den vor ihm erschienenen Arbeiten von Sehaller, Dutroehet, J. Müller wurde der galvanische Strom angewendet, der eine starke, chemische Wirkung besitzt. Rollett $(22,23)$ bediente sich einer Leydener Flasche, deren Fläche der inneren Belegung 493,14 qdem.; die Entladung fand jede 21/2 - 3 Minuten statt und die Funkenlänge betrug $20 \mathrm{~mm}$. Er fand, daß nach einer gewissen Zahl von Entladungen das Blut, durch welches der Strom hindurohging, lackfarbig wurde. Die roten Blutkörperchen wurden zerstört. Endlich untersuchte Rollett (24) das Verhalten verschiedenartigen Blutes zum elektrisehen Strom und die Veränderungen seiner Widerstandsfähigkeit bei Zusatz ron Salz und Zuckerlösungen. Die Elektrizitätsquelle blieb dieselbe. Seine überaus interessanten Ergebnisse, daß das Blut verschiedener Tiere verschiedenen Widerstand dem elektrischen Strome gegenüber besitzt, daß der Zusatz von Salzen und Zucker diese Resistenz je nach der Konzentration des beigemengten Stoffes in geringerem oder größerem Grade erhöht, können aber nicht allein den unmittelbaren Wirkungen des elektrischen Stromes zugeschrieben werden. Denn in allen seinen Versuchen, lieferte die Leydener Flasche keinen :streng gleichmäßigen Wechselstrom. Infolgedessen way bei ihm, wie er selbst in seiner Arbeit (23) (Seite 360) binweist, eine Elektrolyse, die er bemüht war zu vermeiden, dennoch vorhanden, wenn auch in sehr schwachem Grade, und es traten bei der Elektrolyse des Blutplasmas solch starke chemische Agentien 
auf, wie Chlor in statu nascendi, Ätznatron und andere Ätzlaugen, von den schon geringe Quantitäten genügen, um die roten Blutkörperchen zu zerstören. Ebenso wird auch wahrscheinlich der größere Widerstand des Blutes bei einem Zusatz von Salz und Zuckerlösungen hervorgerufen durch seinen größeren Widerstand den chemischen Produkten der Elektrolyse gegenüber.

Teilweise in derselben Richtung arbeiteten auch $S \mathrm{ch}$ orfen or th 25), der die Veränderung der Resistenz der roten Blutkörperchen in ihrem Verhalten zu verschiedenen chemischen und physikalischen Agentien (Elektrizität, Kälte, Wärme, Galvanisieren, Salzen, Äther und Alkohol) bei Zusatz von Salzen alkalischer Metalle untersuchte. Das allgemeine Ergebnis dieser Untersuchungen ist, daB die dem Blute beigemengten Salze die Resistenz des Blutes in Bezug auf physikalische Agentien erhöben, in Bezug auf chemische erniedrigen. Bei seinen Untersuchungen benutzte anch er allem Anscheine nach den Strom einer Leydener Flasche, jedoch ist weder die Stromstärke noch die Spannung angegeben, und was noch wichtiger ist, es ist nicht angegeben, ob eine Elektrolyse stattfand oder nicht. Das Verfahren Rollett's, nur unbedeutend verändert, benutzte auch Lacker (26). Fr bediente sich auch einer Leydener Flasche (die Höhe der inneren und ăußeren Belegung betrug $11 \mathrm{~cm}$ ) und bestimmte nach der Zahl ihrer Entladungen, die geniigten, um das Blut lackfarbig zu machen, seine specifische Resistenz. In seiner Arbeit beschreibt der Autor die bei den Experimenten benutzten Einrichtungen so unvollständig, daß es nicht klar wird, ob er eine konstante Stromart verwendete, oder ob eine oscillatorische Entladung stattfand. Auch gibt er selbst nicht an, ob Erscheinungen der Elektrolyse eintraten.

Endlich wandte auch $\mathrm{B}$ uffa (27) im verflossenen Jahre den elektrischen Strom zur Bestimmung der Resitsenz der roten Blutkörperchen an.

Er vermengte das Blut mit einer $0.7 \%$ Chlornatrium-Lösung und teilte es in 2 Portionen. In der einen von ihnen wurde mit Hilfe eines Chromokytometers von Bizzozero die Quantität der roten Blutkörperchen bestimmt; die andere Portion wurde der Wirkung eines galvanischen Stromes unterworfen und dann wiederum auf dieselbe Weise die Quantität der roten Blutkörperchen bestimmt, die sich nach der Wirkung des elektrischen Stromes verringerte. Der Strom wurde einem galvanischen Elemente entnommen und, obwohl die Konstruktion des sehr komplizierten Apparates darauf gerichtet war, die Eektrolyse zu beseitigen, wird dies Ziel mit dem Apparate B uffa's nicht erreieht. Der Autor weist selbst darauf hin, daß er eine komplizierte Erseheinung vor sich hatte, da die Wirkung der Elektrizität mit derjenigen der Elektrolyse sich vereinte.

Wie aus der angeführten Übersicht der Arbeiten zu ersehen ist, ist die Literatur uber den Einfluf des elektrischen Stromes auf die geformten Elemente des Blutes sehr unbedeutend. Zudem leiden. 
sie alle an einem gemeinsamen Mangel: Es wurdc bei allen Untersuchungen entweder der galvanische, kanstante Strom, oder der Strom einer Leydener Flasche benutzt, und daher war auch immer eine Elektrolyse vorhanden. Außerdem ist anch bei keinem der Autoren die Temperatur des Mediums angegeben und nicht bemerkt, ob diese sich während des Experimentes unter dem Einfluß des Durchfießens des elektrischen Stromes änderte. Die allmälige Erwärmung: des Blutes bis zu $52{ }^{\circ} \mathrm{C}$ ist aber schon imstande, die roten Blutkörperchen vollständig zu zerstören und das Blut lackfarbig zu machen (Schulze 28).

Ich stellte mir die Aufgabe, die unmittelbare Wirkung des elektrisehen Stromes selbst, unter Ausschluß der Elektrolyse und Erwärmung, zu untersuchen. Um der Elektrolyse vorzubeugen, ist es notwendig, sich eines Wechselstromes zu bedienen, bei dem die Unterbrechungen genügend häufig und gleichmäßig sind. Auch unter diesen Bedingungen findet eigentlich doch noch eine Elektrolyse statt; da aber der Strom fortwährend seine Richtung ändert und die gleiche Zeit in jeder Richtung fleßt, so heben sich die chemischen Wirkungen auf und es bleiben merkliche Wirkungen der Elektrolyse aus, d. b. der chemische Zustand des Mediums bleibt unverändert. Die andere störende Nebenwirkung, die Erwärmung, läßt sich sehr leicht beseitigen, indem das Gefäß, durch das der Strom fließt, fortwährend abgekühlt wird.

Die weiter unten zu beschreibenden Experimente sind von mir mit freundlicher Bewilligung des Herrn Prof. S. J. Tereschin im physikalischen Institut der Medizinischen Militär-Akademie ausgeführt worden, wo ich die verschiedensten Elektrizitätsquellen benutzen konnte.

Das Verfahren meiner Experimente bestand im folgenden.

In ein Gläschen mit parallelen Wänden von einem Volumen von $4 \mathrm{ccm}$ mit einem regelmäßigen Quadratboden von $1 \mathrm{qcm}$ wurde $1,5 \mathrm{qcm}$ einer Lösung eingegossen, zu der darauf das Blut hinzugefügt wurde. Das durch einen Stich aus dem weichen Teile eines Menschenfingers oder Kaninchenohres (die mit Quecksilbersublimat, Spiritus und Äther abgewaschen waren) erhaltene Blnt wurde mit einer Fleisch'schen Kapillare von einem Volumen von 8,2 Kubikmillimeter aufgenommen und sofort in das Gläschen übergefubrt. Als Elektroden dienten zwei Platinplatten, von denen die eine auf den Boden des Gefäßes gesetzt wurde, die andere jedoch die Oberfläche der Flüssigkeit berührte. Die beiden quadratförmigen Platten paßten so genau in den Raum des Gläschens, daß sie auf eixem beliebigen Niveau von selbst sich hielten. Die Elektroden wurden in Form von Platten aus dem Grunde gewählt, um einerseits die Berührungsfläche der Flüssigkeit zu vergrößern, 
andererseits um die Erscheinung der Polarisation der Elektroden zu vermeiden. Von den beiden Platten gingen Platindrähte aus, an denen dann die Leiter zur Elektrizitätsquelle befestigt waren. Der Draht, der von der untern Elektrode ausging, war in einem dünnen Glasröhreben untergebracht und infolgedessen vollständig isoliert. So befand sich die Flïssigkeit mit den Blutelementen zwisehen den beiden Platteñ, zwischen denen der Strom floß. Bekanntlich macht das Blut bei einer Verdünnung von 1:200 (ich babe eine größere Verdünnung nicht angewendet) die Flüssigkeit vollständig undurchsichtig, solange die Blutkörperchen nicht zerstört sind, je nach der Zerstörung dieser wird die Lösung mehr und mehr durchsichtig. Zur Bestimmung des Klarwerdens benutzt man in unserer Klinik ein Verfahren, dessen auch ich mich bediente: Hinter dem Gläsehen mit der Blutlösung werden Lettern aufgestellt $(0,6$ D. nach den Tabellen zur Bestimmung der Sehschärfe), und beim Klarwerden der Flïssigkeit tritt zuletzt die Möglichkeit ein, die Sehrift durch die Flï̇sigkeitsschicht im Gläschen zu lesen. Bei den Experimenten benutzte man entweder Tageslicht (hauptsächlich), oder eine elektrische Lampe von 25 Kerzen. Eine jede von mir aufs neue verwendete Elektrizitätsquelle priffte ich zuvor, $o b$ bei ihr Elektrolyse stattfinde. $\mathrm{Zn}$ diesem Zwecke füllte man ein Gefäß mit einer Chlornatr.-Lösung oder mit angesäuertem Wasser und mit Hilfe der oben beschriebenen Elektroden wurde der zu untersuchende Strom durch die Flüssigkeit hindurchgelassen. Das gänzliche Fehlen von Gasbläschen, die aus der Lösung austreten, wies unzweifelhaft darauf hin, daß keine Elektrolyse vorhanden war. Anfangs nahm ich als Medium zur Vermengung mit dem Blute eine 0,9\% Chlornatr.Lösung, in welcher eine merkliche Zerstörung der roten Blutkörperchen selbst nach 24-28 stündigem Verbleiben nicht stattfand.

Anfänglich benutzte ich den Strom eines Rumkorf'schen Induktionsapparates. Den Strom bezog man von 3 hintereinander geschalteten Akkumulatoren von einer elektromotorischen Kraft von 2 Volt, der Apparat mit einem Maximalfunken von $30 \mathrm{~cm}$ lieferte bei unseren Experimenten einen Funken von ungefähr $2 \mathrm{~cm}$. Der Apparat mit der Blutmischung und dem $0,9 \%$ Chlornatrium wurde in die sekundäre Kette eingeschaltet.

Ich halte es für notwendig zu bemerken, daß wie in diesem Falle, so auch in allen folgenden eine ganze Reihe von Experimenten ausgeführt wurden, da aber das Resultat stets ein und dasselbe blieb, so führe ich sie nicht einzeln an, sondern nur das allgemeine Ergebnis. Der Strom des oben beschriebenen Induktionsapparates durchfioß das Blut im Laufe von 10-30 Minuten; während der ganzen Zeit blieb die Flüssigkeit gleichmäßig trüb, wie am Beginn des Experiments; eine jede Erwärmung blieb aus and die roten Blutkörperchen wurden nicht zerstört.

In der Vermutung, daß die Spannung des Induktionsstromes nicht groß genug sei, um die roten Blutkörperchen zu zerstören, nahm ich den größten, in der Akademie befindlichen Rumkorfschen Induktions- 
apparat. Dieser Apparat (er befindet sich im Radiographischen Kabinett des klinischen Militärhospitals) hat eine Maximalfunkenlänge von $60 \mathrm{~cm}$. Der Strom von 100 Volt Spannung rührte von einer Akkumulatorenbatterie her und ging durch einen Wehnelt-Unterbrecher in die primäre Spule; die Enden der sekundären Spule wurden mit den Platinelektroden meines Apparates verbunòen. Auch diesmal erhielt man negative Resultate, obgleich der Strom 10-20 Minuten flof und die Spannung des elektrisehen Stromes an den Enden der sekundären Spule bei diesem Apparat sehr bedeutend war.

Die oben beschriebenen Tatsachen befinden sich augenseheinlich im Widerspruch mit den Experimenten 0 wsjanikows1), der bei Anwendung des Apparates von Dubois-Reymond unter dem Mikroskope sehr starke Veränderungen in den Blutkörperchen sah, die mit ihrer völligen Zerstörung endeten. Der Grund der Abweichung meiner Resultate von den seinigen liegt allem Anscheine nach in der Anordnung der Experimente. Der vom erwähnten Autor beschriebenen mikroskopischen Technik entsprechend, dienen als Elektroden bei seinem Apparate 2 außerordentlich dïnne und dazu noch an den sich gegenseitig zugewendeten Enden zugespitzte Stanniolblättchen; außerdem war auch die Entfernung zwischen diesen Elektroden sehr klein. Folglich fließt der Strom zwischen 2 Spitzen, seine Dichte wird sehr bedeutend sein, und daher mul sich unvermeidlich Wärme entwickeln, und zwar eine recht bedeutende. Gewiß kann diese Temperaturerhöhung infolge der geringen Quantität der Fliissigkeit thermometriseh nicht bestimmt werden, aber sie muß existieren, und unter ihrem Einfluß findet wahrsobeinlich die rom Autor beschriebene Veränderung des Blutes statt. Anßerdem tritt, wie ich es bei meinen Experimenten beobachten konnte, zuweilen bei den Apparaten von DuboisReymond eine Elektrolyse auf, wie es seheint infolge der ungleichmäßigen Tätigkeit des Unterbrechers. Eine Bestätigung der oben erwähnten Vermutungen kann man in den folgenden, von mir ausgeführten Experimenten sehen.

Zu zwei mit gleicher Menge ein und derselben $0,9 \%$ Chlornatr. Lösung gefüllten Gläschen fügt man gleichzeitig je $8,2 \mathrm{cbmm}$ gleichen Blutes hinzu; durch das eine von ihnen fließt der Strom eines Rumkorfschen Induktionsapparates hindurch. Jede 5 Minuten nabm man je einen Tropfen aus dem einen und dem andern Gläschen und untersuchte sie unter dem Mikroskop; man nahm keine Veränderung an den Präparaten wahr, ungeachtet der $30-40$ minutenlangen Einwirkung: des Stromes. Wenn also die Elektroden breit sind und die Dichte des Stromes gering ist, findet keine Erwärmung statt, und es bleibt jede Änderung der roten Blutkörperchen aus. 
Wie zu erwarten war, erhielt man ebensolehe negative Resultate auch bei der Wirkung des sinusoidalen Stromes. Der von mir benutzte Apparat ist vom Lehrer der Minenoffizierschule in Kronstadt A. L. Popow konstruiert; von ihm und Dr. P. J. Jschewsky ist auch der Beweis erbracht worden, daß der Strom dieses Apparates tatsächlich ein rein sinnsoidaler ist (30).

Alle oben erwähnten Apparate liefern einen Strom, wenn auch von großer Spannung, die in einigen Apparaten bis zu hunderttausenden Volt steigt (ein Funke von $60 \mathrm{~cm}$ ), so doch von einer sehr geringen Stärke, und daher mußte man, nachdem man mit ihnen negative Resultate erhalten hatte, sich bemühen die Stromstärke zu vergrößern.

Leider war es nicht möglich, den starken Wechselstrom einer Haus'schen Maschine, die zur elektrischen Beleuchtung der Akademie dient, za benutzen, da dieser Strom eine sehr starke Elektrolyse hervorrief. Es ist bekanut, daß, indem man den Widerstand der sekundären Spule des Induktionsapparates verringert, seine Stromstärke vergrößert. Dies läßt sich erreichen, indem man die beiden Spulen des Apparates aus diesem Draht gleichen Durchmessers herstellt. Einen solchen Apparat verwendete ich auch bei meinen späteren Experimenten. Die Stromquelle bildete eine Akkumulatorenbatterie von der elektromotorischen Kraft von 60 Volt; der Strom ging durch einen Rheostat, Ampèremeter, Wehnelt-Unterbrecher in der primären Spule, die Enden der sekundären Spule wurden mit meinem Apparat verbunden. Die Stärke des konstanten Stromes in der primären Kette schwankte um 10 Ampère; der Wechselstrom der sekundären Spule brachte einen etwas weniger als einen Millimeter dicken Platindraht zum Glühen, d. b. der Strom war genïgend stark.

Merkliche Erscheinungen der Elektrolyse traten nicht ein, folgich hatten wir einen gleichmäßigen Wechselstrom. Wurde dieselbe Mischung: von 1,5 com einer $0,9 \%$ Chlornatriumlösung und $8,2 \mathrm{cmm}$ Blut der Wirkung des Stromes ausgesetzt, dann waren die Erscheinungen andere, da eine Wärmewirkung des elektrischen Stromes eintrat. Dabei wurden die roten Blutkörperchen zerstört und tauchten in Gestalt von Schaum auf der Oberfläche der Flüssigkeit auf, sobald sich die Flüssigkeit im Gläschen genügend stark erwärmt hatte; man brauchte aber nur dureh Einschaltung eines Rheostaten die Stromstärke zu verringern, damit sogleich die Erwärmung und die Zerstörung der roten Blutkörperchen langsamer von statten ging, oder überhaupt nicht mehr beobachtet wurde. Um noch deutlicher zu zeigen, daß die Wärme im gegebenen Falle als Zerstörerin der roten Blutkörperchen wirkt, kühlte ich bei derselben Aufstellung 
des Experimentes das Gläsehen mit der Mischung vom Blut und der Salzlösung ab. Zu diesem Zwecke tauchte ich es in ein Gefäß mit Schnee oder in ein Gefäß mit kaltem Wasser, welches gentigend oft gewechselt wurde. Tatsächlich zeigte es sich, daß man, wenn nur die Flüssigkeit im Gläschen kalt blieb, den stärksten Strom hindurchgehen lassen und das Blut der Wirkung des Stromes 10-30 Minuten aussetzen konnte, ohne die Blutkörperchen zu zerstören, während bei derselben Stromstärke, ohne Abkühlung, eine 5 minutige Wirkung zu ihrer völligen Zerstörung genügte. Und so erscheint bei großer Stromstärke als einzige Zerstörerin der roten Blutkörperchen die Wärme. In dieser Serie von Experimenten prüfte ich auch mit Hilfe des Mikroskops meine Beobachtung und konnte beim Ausbleiben der Erwärmung keine Veränderung der roten Blutkörperchen bemerken. Folglich ist weder die Spannung noch die Stromstärke an und für sich im stande, die geformten Elemente des Blutes in einer isotonischen $(0,09 \%$ Chlornatrium-Lösung zu zerstören.

Außerdem verwandte ich noch eine Art des elekrischen Stromes, und zwar eine oscillatorische Entladung von großer Häufigkeit.

Diese Art des elektrischen Stromes erhielt man durch eine Kombination von Apparaten, die von der Fabrik $\mathrm{H}$ eff in Paris konstruiert wurden. Indem ich zur näheren Kenntnisnahme von den Apparaten auf die Dissertation des Herrn Dr. P. J. J s che wsky (31) verweise, erwähne ich bloß, daß ein Strom von 100 Volt Spannung vom Elektrizitätswerke durch einen Transformator dieses Systems sich in 15000 Volt verwandelte; in der primären Kette betrug die Stromstärke 5 Ampère (bei dieser Stärke erhält man den größten Leistungseffekt); die große Solenoide wurde bei meinen Experimenten ausgeschlossen, das eine Ende der kleinen Solenoide wurde mit der Erde verbunden, das andere mit einem Oudin'schen Resonator; von dort aus floss der Strom zu meinem Apparat. Unter solchen Bedingungen erhält man durch die oscillatorische Entladung gegen $1 / 2$ Million Schwingungen in einer Minute; es tritt weder die Elektrolyse, noch Temperaturerhöhung ein.

Das Blut, welches auf die oben erwähnte Weise mit einer isotonischen Chlornatrium-Lösung vermengt war, wurde der Wirkung: des Stromes im Laufe von 10-15 Minuten ausgesetzt, ohne daß irgendwelche Zerstörungserseheinungen oder Veränderungen in den roten Blutkörperchen eingetreten wären. Also erwiesen sich alle mir zur Verfügung gestellten Arten des elektrischen Stromes, sobald sie weder eine Elektrolyse, noch eine Erwärmung hervorriefen, als unfähig, die roten Blutkörperchen in einem isotonischen Medium zu zerstören. 
Davon ausgehend, daß in den roten Blutkörperchen sich Eisenverbindungen befinden, untersuchte ich beilänfig die Wirkung des elektromagnetischen Feldes auf sie. Eine Mischung von Blut wurde zwischen den Polen eines Faraday'schen Elektromagneten untergebracht, zu dem ein elektrischer Strom (von 33 Volt Spannung) von einer Akkumulatorenbatterie führte. Auch in diesem Falle erhielt ich vollständig negative Resultate.

Wie ist eigentlich dieses indifferente Verhalten der roten Blutkörperehen gegenWechselströme zu erklären? Ist es von den Eigenschaften der Körperchen an und für sich oder von denjenigen äußeren Bedingungen abhängig, unter denen der elektrisehe Strom seine Wirkuug auszuiuben hat? Die roten Blutkörperchen befinden sich im Plasma, d. h. in einer Salzlösung, und in meinen Experimenten wurden sie dazu noch mit $0,9 \%$ Chlornatrium vermengt; die Salzlösungen sind aber als gute Leiter des elektrischen Stromes bekannt. Man könnte voraussetzen, daß der Strom infolge der großen Leitungsfähigkeit des Mediums den leichtesten Weg, sozusagen ohne in die Blutkörperchen einzudringen, wählt. Um diese Frage zu erläutern, muß man die Leitungsfähigkeit der 0,9\% Chlornatrium-Lösung mit derjenigen der roten Blutkörperchen vergleichen.

Koeppe (32) fand, indem er einen Niederschlag der roten Blutkörperchen des Pferdes herbeifuhrte, daß ihre Leitungsfähigkeit, in praktischen Elektrizitätseinheiten ausgedruickt, von $6,2 \times 10^{-8}$ bis $6,8 \times 10^{-8}$, d. h. durchschnittlich $6,5 \times 10^{-8}$ gleich ist, oder ihr Widerstand ungefähr 15 Millionen $\mathrm{Ohm}$ beträgt. $\mathrm{Bu}$ garsk y nnd Taugel (33), die dieselben Untersuchungen sorgfältiger ausgefübrt haben, fanden, daß die Leitungsfähigkeit der roten Blutkörperchen, die durch Z entrifugieren vom Plasma isoliert wurden, nur von $1,62 \times 10^{-8}$ bis $2,44 \times 10^{-8}$, im Durchschnitt 2,03 $\times 10^{-8}$ gleich ist, oder daß der Widerstand gegen 50 Millionen $\mathrm{Ohm}$ beträgt. Wenn wir uns vergegenwärtigen, dab die Leitungsfähigkeit des Dielektrikums des destillierten Wassers $0,048 \times 10^{-8}$ (prakt. Einheiten) gleich ist, so sehen wir, daß die roten Blutkörperchen ihrer Leitungsfähigkeit nach an die Nichtleiter heranrücken.

Es ist durchaus möglich, daß die oben angeführte recht wesentliche Differenz zwisehen dem destillierten Wasser und den roten Blutkörperchen auf die Unvollkommenheit des Verfahrens zurückzuführen sei. Es ist möglich, daß die roten Blutkörperehen an und für sich den elektrischen Strom absolut nicht leiten, und dab die für sie von den Autoren gefundene Leitungsfähigkeit von der Beimengung der Reste des Blutplasmas herrührt, umsomehr als die Leitungsfähigkeit sich rasch verringert, wenn ihre Trennung vom. Plasma sorgfältiger ausgefübrt wird. (Siehe Bugarsky). Andererseits ist die Leitungsfähigkeit der Salzlösungen sehr groß. 
Eine $0,57 \%$ Chlornatrium-Lösung im Wasser besitzt eine Leitungsfähigkeit in bezug auf Quecksilber nach den Tabellen von Landolt (34) von $8650 \times 10^{-8}$, was in praktischen Elektrizitätseinheiten ausgedrïckt, $916900 \times 10^{-8}\left(9169 \times 10^{-6}\right)$ gleich ist; die Leitungsfähigkeit der von uns benutzten isotonisehen $0,9 \%$ Chlornatrium-Lösung ist jedoch ungefähr $1350000 \times 10^{-8}\left(135 \times 10^{-4}\right)$ prakt. Einheiten gleich; wenn man aber von hier aus zum Widerstande ïbergeht, so wird er ungefähr $74 \mathrm{Ohm}$ entsprechen. Folglich sehen wir, dab die Leitungsfähigkeit der roten Blutkörperchen tausende von Malen geringer ist als die der Salzlösungen, in denen sie sich befinden.

Um den Widerstand des Mediums zu erhöhen, nahm ich statt der isotonischen $\mathrm{NaCl}$ Lösung eine fast auch isotonische Lösung von Rohrzucker von $5 \%$ (H a mburger) (9). Zuerst fand ich gemeinschaftlich mit dem Assistenteñ des physikalischen Institutes A. N. Ge orgiewsky, daf der Widerstand dieser Lösung nicht kleiner als 11000 Ohm ist, folglich ist die Leitungsfähigkeit nicht größer als $9090 \times 10^{-8}$ (prakt. Einheiten). Also setzen wir, indem wir anstatt der $0,9 \% \mathrm{Cl} \mathrm{Na}$ Lösung eine $5, \check{\partial} \%$ Rohrzuckerlösung gebrauchen, die Leitungsfähigkeit des Mediums gegen $150 \mathrm{Mal}$ herab.

Bei dieser Anderung des Verfahrens führte ich eine Reihe von Experimenten mit den oben erwähnten Elektrizitätsquellen aus und wiederum erhielt ich in allen Fällen dieselben negative Resultate. Weder unter dem Mikroskope, noch mit blobem Ange konnte ich irgend welche Veränderungen der roten Blutkörperchen beobachten, mochtensie der Wirkung des elektrischen Stromes ausgesetzt sein oder nicht.

Aus allen angeführten Experimenten folgt, daß der Weehselstrom ohne die ihn begleitenden Erscheinungen, der Elektrolyse und der Erwärmung, keine merkliche Wirkung auf die roten Blutkörperchen ausübt, daß die Stärke und Spannung des Stromes an und für sich, wie auch der Widerstand des Mediums hier wirkunglos bleiben. Rührt dieses von einer besonderen spezifischen Resistenz der roten Blutkörperchen dem elektrischen Strome gegenüber her, oder leiten sie einfach absolut nicht den Strom, und erfahren daher nicht seine Wirkung - dieses kann ich auf Grund meiner Arbeit und der literarischen Quellen nicht sagen. Letztere Vermutung wird übrigens teilweise durch die erwähnten Experimente ron Koeppe, Bugarsky und Taugel gestützt.

Zum Sehluß habe ich Herrn Professor S. J. Tereschin und seinen Assistenten Herren Dr. A. N. Orlow und A. N. Georgiewsky meinen innigsten Dank für ibre stete Bereitwilligkeit, mir mit Rat und Tat zur Seite zu stehen, auszusprechen.

Gleichzeitig danke ich auch Herrn Dr. P. R. Jschewsky für seine Hilfe bei meinen Experimenten mit Entladungsströmen. 


\section{Literatur.}

1) Prof. M. W. Janowsky. Über die Resistenz der roten Blutkōrperchen. Berichte der Kaiserl. Mediz.-Militär-Akademie. Oktober 1900. S.133.

2) Lang. Über die diagnostische Bedeutung der Resistenzerhöhung der roten Blutkörperchen und anderer Veränderungen beim Magenkrebs. Dissert. St. Petersburg 1901.

3) I wanow. Über das Verhältnis der Veränderungen der Resistenz zur Menge der Mineralbestandteile der roten Blutkörperchen. Dissert. St. Petersburg 1901.

4) Itin. Über die Änderung der Resistenz der roten Blutkörperchen unter dem Einfluß des Gebrauches von Borschower Wasser (Ekaterinoslawquelle). Dissert. St. Petersburg, 1902.

5) Landois-Eulenburg. Realencyclopädie B. III. 2. Aufl. (cit. nach Urcelay 35).

6) Duncan. Comptes rendus de la soc. de Biologie, 1895 Janvier (cit. nach Urcelay 35).

7) Janowsky. Materialen zur Frage über die pathologische Bedeutung der Resistenzerhöhung der roten Blutkörperchen. Berichte der Kaiserl. Mediz.-Militär-Akademie. Januar 1901.

8) Vaquez. De methodes propres à evaluer la resistence de glolules rouges du sang. Semaine modicale, 1898.

9) Hamburger. Über den Einfluß chemischer Verbindungen auf Blutkörperchen. Arch. für Physiologie. 1886 (cit. nach Janowsky 1).

10) M osso. Resistance de globules rouges. Arch. Italiennes du Biolog. VIII. p. 257 (cit. nach Urcelay 35).

11) Nedrigailow. Vergleichende Untersuchungen der Resistenz der roten Blutkörperchen bei Unterleibstyphus im Verhältnis zu Chlornatrium und Cblorḱaliumlösungen. Dissert. Petersburg 1899.

12) Poschin. Zur Frage über die Resistenz des Blutes bei Chlorose und Anämie. Dissert. St. Petersburg 1900.

13) Malassez. Les premieres recherches sur la resistance des globules rouges du sang. Comptes rendus de la Soc. de Biologie. Janv. 1905, (cit. nach Urcelay 35 ).

14) Chanel. Compte rendu des travaux du laboratoire de clin. méd. de la facul. de médic. de Lyon. Revue mensulle de medicine et 'de chir. 1880.

15) Mauca. Influenza della cocaina sulla resistenza du globuli rossi del sangue. Lo sperimentale. $1894, \mathrm{Nr}$. 48 (cit. nach Janowsky 1).

16) Maragliano et Castellino. De la nécrobiose lente de globules rouges à l'état normale et pathologique. Arch. Ital. de Biol. 1893 p. 55 .

17) London. Zur Lehre von den Hämolysinen. Dissert. St. Petersburg, 1800.

18) Schkljarewitz. Das biologische Verfahren zur Resistenzbestimmung der roten Blutkörperchen. Klinische Zeitung von Botkin. 1901. (Sonderabdruck.)

19) Bjelonuwsky. Ein neues Verfahren zur Resistenzbestimmung des Blutes. Klinische Zeitung von Botkin. 1902, Nr. 1.

20) Maragliano. Berliner klinische Wochenschrift. 1887, Nr. 43 (cit. nach Janow $8 \mathrm{ky} \mathrm{1)}$. 
21) Battazzi und Ducceschi. Arch. Ital. de Biologie. 1896. (cit. nach I wanow 3).

22) Rollet. Versuche und Beobachtungen am Blut. Sitzungsber. der Akad. der Wissenschaft. 1863. Wien. XLVII. B. II. Abt.

23) Derselbe. Über die Wirkung des Entladungsstromes auf das Blut. Sitz* ungsber. d. Akad. d. Wissensch. XLVII. B. II. Abt.

24) Derselbe. Über die Wirkung, welche Salze und Zucker auf die roton Blutkörperchen ausüben. Sitzungsber. d. Akad. d. Wissenschaft LXXXIV. B. III. Abt. p. 157. 1882.

25) Schrofenorth. Über den Einfluß der Salze auf die Lösung der roten Blutkörperchen durch verschiedene Agentien. Centralblatt f. d. med. Wissenschaft. 1884, Nr. 50, p. 907.

26) Laker. Über eine neue klinische Blutuntersuchungsmethode. Wiener med. Presse 1890, pag. 1375.

27) Buffa. Resistenza dei globuli rossi del sangue. Archivio per la scienze mediche V. XXV, Nr. 10, p. 187, 1901.

28) Schülze. Cit. nach d. Encyklop. der Mediz. Wissensch. T. IX, Artik. 162.

29) Landowsky und Owsjannikow. Grundlagen zum Studium der mikroskopischen Anatomie des Menschen und der Tiere. T. I, Kap. XVI. Art. $12 \%$.

30) Ischewsky. Über sinusoidale Ströme.

31) Ischewsky. Über den Einfluß des elektromagnetischen Feldes auf den Organismus, Dissert. St. Petersburg. 1900.

32) H. Koeppe. Die Volumensänderung der roten Blutscheiben in Salzlösungen. Arch. f. Physiol. 1899, p. 515.

33) Bugarski und Tangl. Centralblatt f. Physiol. 1897 (cit. nach Koeppe 32). 34) Landolt $\mathfrak{u}$. Börnstein. Physikalisch-chemische Tabellen. 1894, p. 481.

35) Uxcelay. De la résistance des globules rouges. Thése. Paris. 1895. 